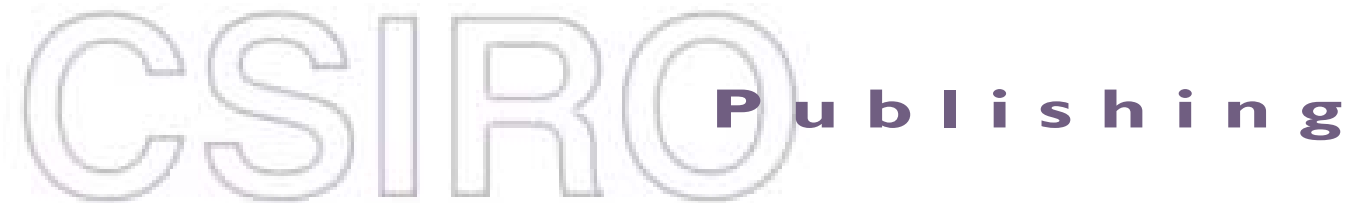

\section{Publications of the Astronomical Society of Australia}

Volume 19, 2002

(C) Astronomical Society of Australia 2002

An international journal of astronomy and astrophysics

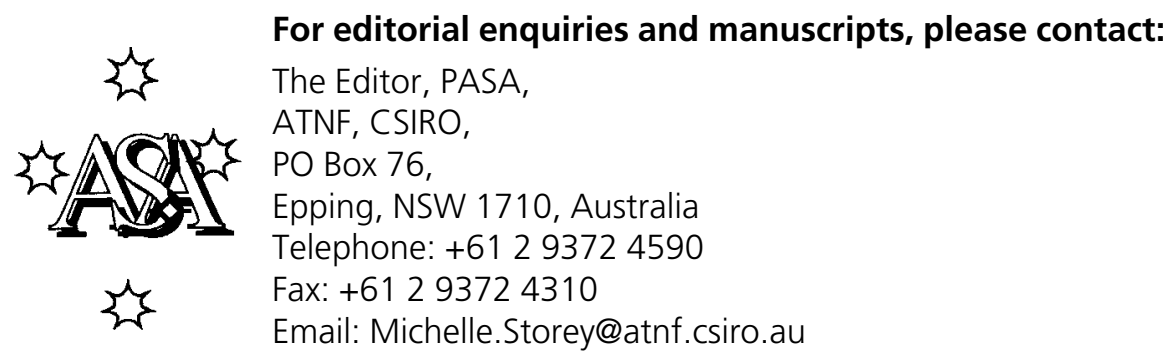

For general enquiries and subscriptions, please contact: CSIRO Publishing PO Box 1139 (150 Oxford St)

Collingwood, Vic. 3066, Australia

Telephone: +6139662 7666

Fax: +61 396627555

Email: publishing.pasa@csiro.au

C S I RO

PUBLISHING Published by CSIRO Publishing

for the Astronomical Society of Australia

www.publish.csiro.au/journals/pasa 


\title{
Intraday Optical Variability of S5 0716+714
}

\author{
Roberto Nesci, Enrico Massaro and Franco Montagni \\ Department of Physics, University La Sapienza, P.le Aldo Moro 2, I-00185, Roma, Italy \\ Roberto.Nesci@uniroma1.it \\ Received 2001 July 25, accepted 2002 May 2
}

\begin{abstract}
We present an optical intraday variability (IDV) study of S5 0716+714 carried out over 52 nights. The source showed detectable variations $(>0.05 \mathrm{mag})$ in $80 \%$ of the nights. Typical variation rates of $0.02 \mathrm{mag} /$ hour have been found to last on average 4 hours, with the average rising rate faster than the decreasing one. A maximum rising rate of $0.16 \mathrm{mag} / \mathrm{hour}$ has been observed. If the luminosity variation is due to jittering in the jet direction, a comoving $d \theta / d t$ rate of $\sim 100 \mathrm{arcsec} /$ hour is required, assuming for the jet $\theta \sim 7^{\circ}$ and $\Gamma \sim 14$.
\end{abstract}

Keywords: BL Lacertae objects: individual (S5 0716+714)

\section{Introduction}

The radio source $\mathrm{S} 50716+714$ is a highly variable BL Lac object, both on timescales of hours (Heidt \& Wagner 1996; Nesci et al. 1998; Giommi et al. 1999; Villata et al. 2000) and on timescales of months (Ghisellini et al. 1997; Raiteri et al. 1999). A firm statistical definition of the properties of its short term variability is a prerequisite to constrain the physical mechanisms responsible for its luminosity variations. We have therefore been undertaking a long term campaign since 1999 to explore the intraday optical behaviour of this source at different luminosity levels.

Being circumpolar already at mid northern latitudes, it can be easily followed for many hours. Its high brightness (typically $R=14$ ) and the presence of several nearby stars of similar brightness and colour allow differential photometry of good accuracy to be readily obtained even with small sized instruments.

We present in this paper the main observational results and a preliminary interpretation, in terms of geometrical effects, of the IDV of this object.

\section{Observations and Data Reduction}

Observations were performed from January 1999 to April 2001 with the $32 \mathrm{~cm} \mathrm{f} / 4.5$ Newtonian reflector at Greve in Chianti (Florence) equipped with a CCD camera with a back-illuminated SITe SIA501A chip. Standard V (Johnson), R (Cousins) or I (Cousins) filters were used. Typical exposure times were 5 minutes reaching a noise level of $0.01 \mathrm{mag}$ or less. Differential photometry with respect to 3 or 4 comparison stars within the field of view

Table 1. Number of observing runs according to their length (upper two rows) and to the source magnitude (lower two rows)

\begin{tabular}{llllll}
\hline Hours & $2-4$ & $4-6$ & $6-8$ & $8-10$ & $10-12$ \\
Runs & 9 & 5 & 11 & 19 & 8 \\
Mag & $<13$ & $13 .-13.5$ & $13.5-14$ & $14-14.5$ & $>14.5$ \\
Runs & 7 & 15 & 16 & 13 & 1 \\
\hline
\end{tabular}

was performed with IRAF/apphot using a 5 arcsec radius aperture. Table 1 gives the distribution of the observing nights according to their length and to the source magnitude.

In total we collected data in 52 nights, for a total of 398 hours (5446 frames), with 38 nights with runs longer than 6 hours. Some of the longer light curves are given in Figure 1. The photometric accuracy is indicated by the magnitude difference of two reference stars, which is also reported in the plots properly shifted to fit into the scale.

\section{Results}

a) Only in 13 out of 52 nights (25\%) did the source remain stable within $0.01 \mathrm{mag}$. If we consider only the runs longer than 6 hours, the fraction of stable nights is $7 / 38$ (18\%), which is statistically compatible with the result from the total sample.

b) We found an equal number of decreasing and increasing light-curve segments.

c) The average value of the increasing variation rate $(0.052 \pm 0.008 \mathrm{mag} / \mathrm{hour})$ is slightly larger than the decreasing one $(0.031 \pm 0.004 \mathrm{mag} /$ hour $)$.

d) There is no clear correlation between the source magnitude and the amplitude of the IDV, although there is an indication that large variations are observed only when $R<13.5$, as can be seen from Figure 2, upper left panel.

e) There is no clear correlation between the source magnitude and the rate of magnitude variation as can be seen from Figure 2, lower left panel.

The distribution of the observed variation rates is shown in Table 2, where the upper limit of each bin is indicated on each column. The median value for the IDV is $0.02 \mathrm{mag} /$ hour.

\section{Discussion}

In the framework of the current models, in which the emission is produced by a relativistic jet, a luminosity change in 

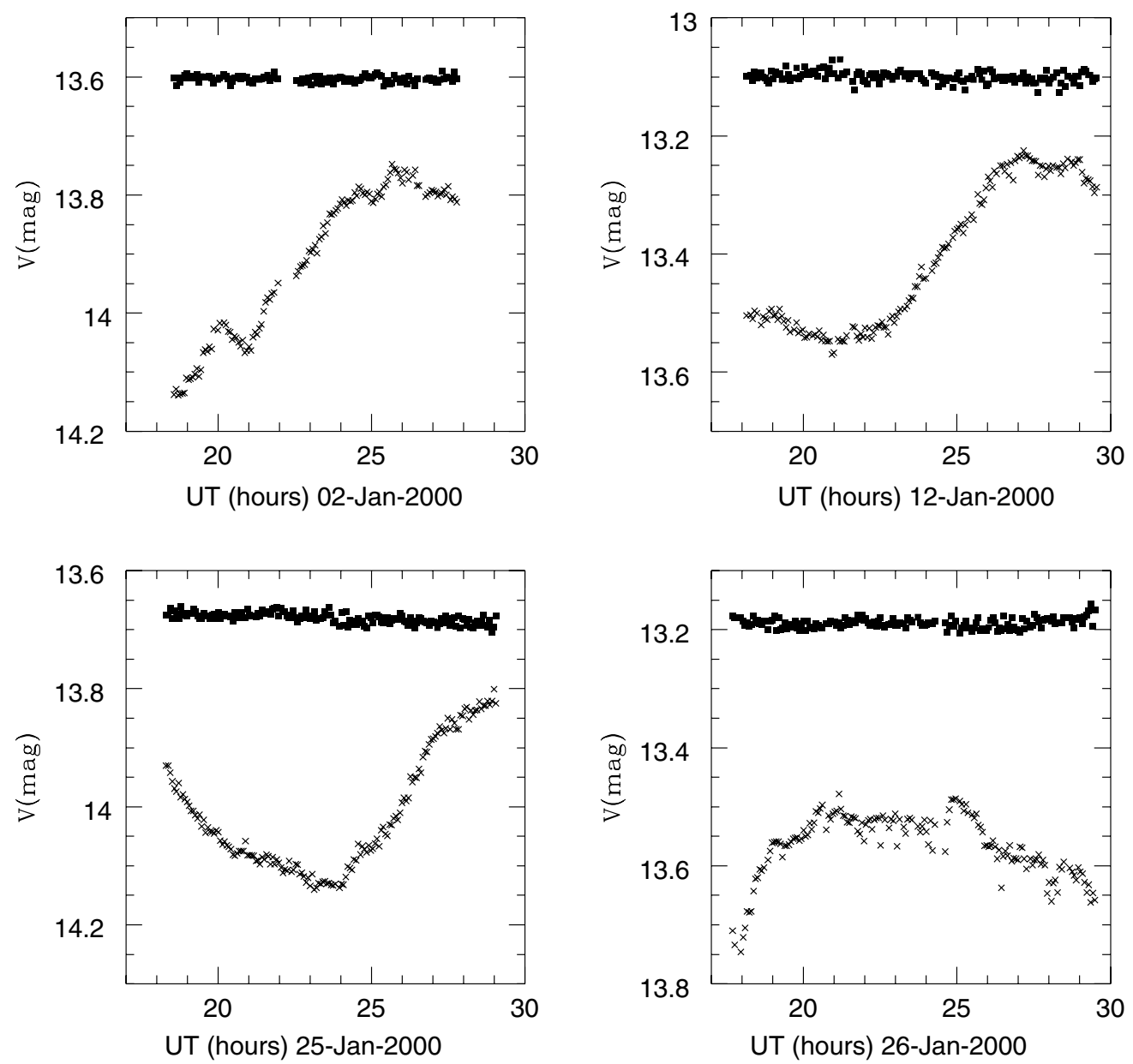

Figure 1 Sample light curves: crosses are S5 0716+714, filled squares are the difference between stars B and C (Ghisellini et al. 1997) shifted to fit into the plotting box.

the optical band may be explained in several ways which basically belong to two classes of models: intrinsic flux changes or geometric effects. We will consider here only the second class.

Assuming that we are looking at a relativistic jet aimed at a small angle $\theta$ to the line of sight and emitting in the optical band by a synchrotron process, and recalling that the Doppler factor $\delta=1 /(\Gamma(1-\beta \cos \theta))$ where $\Gamma=\left(1-\beta^{2}\right)^{-0.5}$ is the Lorentz factor of the bulk motion of the emitting plasma, the relative flux variation for a small change of the jet direction is

$$
\begin{aligned}
d m / d t_{\mathrm{obs}} & =1.086 \times 1 / F_{\mathrm{obs}} \times d F_{\mathrm{obs}} / d t_{\mathrm{obs}} \\
& =(3+\alpha) \beta \Gamma \delta^{2} \sin \theta\left(d \theta / d t_{\mathrm{jet}}\right)
\end{aligned}
$$

where obs and jet label quantities in the observer and in the jet reference frames respectively, and $\alpha$ is the spectral index. The variability rate in mag/hour, expected for a given value $(0.002 \mathrm{rad} /$ hour $)$ of the rate of variation of the $\theta$ angle, is reported in Figure 2, upper right panel, as a function of $\delta$ for some reasonable values of $\theta$. Along the lines of given $\theta$ each cross marks a value of $\Gamma$ from 2 to 21 . It is apparent that very small variations in the direction of the jet (or, better, of the part of the jet mainly contributing to the light observed) are quite sufficient to produce substantial changes in the rate of variation. Changing the adopted value for $d \theta / d t$ produces a linear scaling in the rate of variation leaving the pattern unchanged, so that the figure can be used for any assumed value of $d \theta / d t$.

For a given $\theta$ and $d \theta / d t_{\text {jet }}$ the magnitude variation rate tends to reach a maximum for increasing values of $\Gamma$ and then decreases. Assuming that the maximum value of variation rate detected in our monitoring $(0.16 \mathrm{mag} / \mathrm{hour})$ is an indication of the 'turn-down' position, from the figure we derive $\theta \sim 15^{\circ}, \delta \sim 3.5, \Gamma \sim 7$ as a possible set of parameters. These values are compatible with those suggested by Ghisellini et al. (1993) on the basis of the radio and X-ray fluxes and an assumed redshift $z=0.3$.

Assuming a value $d \theta / d t$ four times smaller ( $\sim 100 \mathrm{arcsec} /$ hour $)$ would imply $\delta \sim 7, \Gamma \sim 14, \theta \sim 7^{\circ}$, rather close to 'typical' assumptions for BL Lac objects.

The IDV episodes detected are short-lived, generally shorter than the length of the night. This may be seen from the examples shown in Figure 1, as well as from the oneweek monitoring of this source on February 1999 by the WEBT collaboration (Villata et al. 2000, their Figure 2). Indeed, if the average IDV trend lasted for 24 hours one would expect a typical difference of $\sim 0.5 \mathrm{mag}$ between consecutive days. This is not the case, as can be seen from 

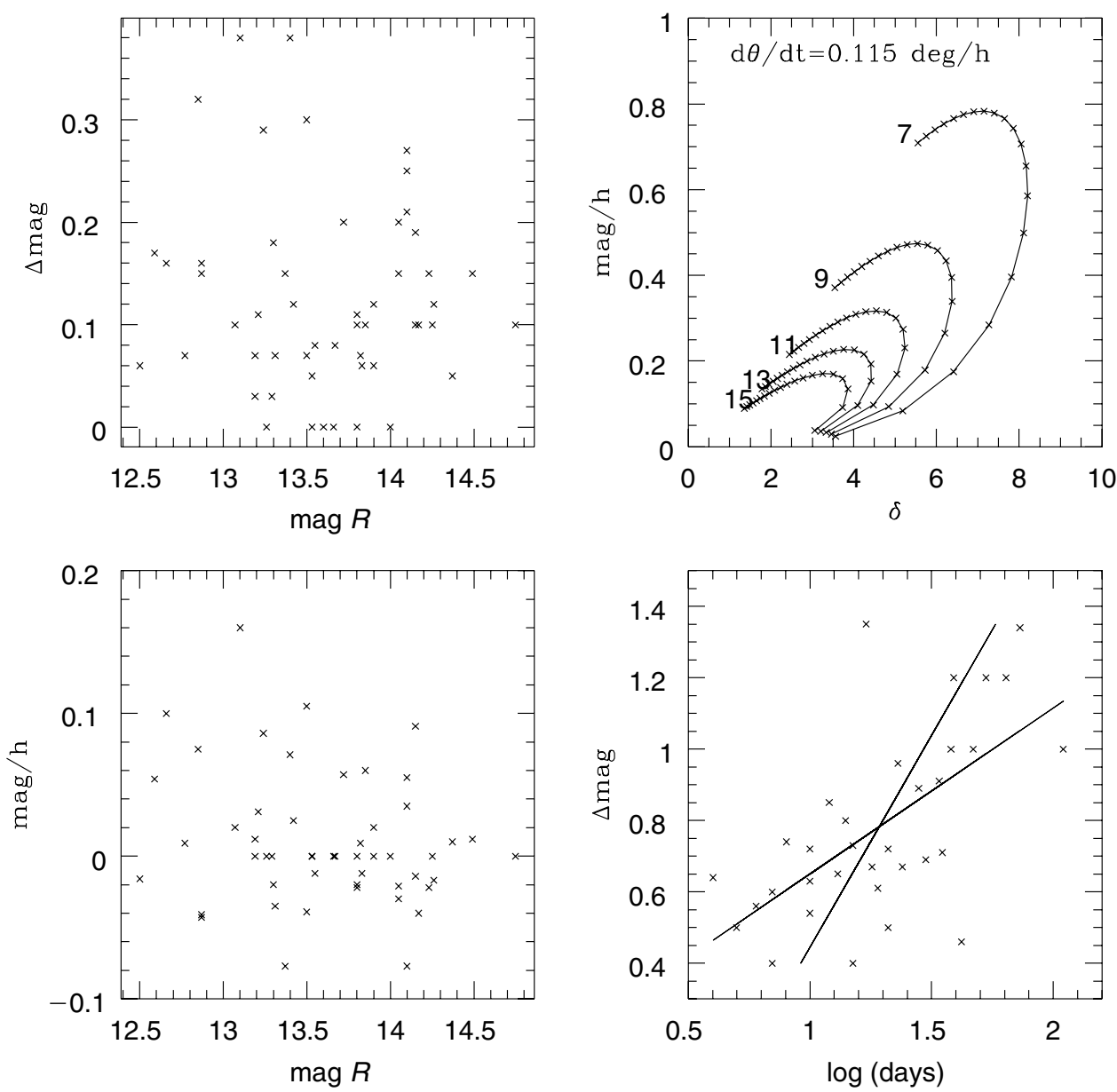

Figure 2 Upper left: intraday overall variation for each observing run vs magnitude. Lower left: intraday variation rate vs magnitude. Upper right: calculated variability rate (mag/hour) vs $\delta$ for different values of $\theta$ (labelled in degrees) and a fixed value of $d \theta / d t$. Crosses along each line of constant $\theta$ mark values of $\Gamma$ from 2 to 21 . A change in the adopted value of $d \theta / d t$ produces a proportional change in the ordinate (variability rate) scale leaving the general pattern unchanged. Lower right: amplitude of the long term 'flares' defined in the text (Section 4) vs flare duration; two best fit lines are drawn, computed using the first or second variable as the independent one.

Table 2. Distribution of the moduli of the variation rate

\begin{tabular}{llllllll}
\hline Mag/hour & $<0.01$ & 0.02 & 0.04 & 0.06 & 0.08 & 0.10 & $>0.10$ \\
Number & 13 & 16 & 7 & 7 & 4 & 4 & 1 \\
\hline
\end{tabular}

Table 3. Distribution of the magnitude difference after 24 hours

\begin{tabular}{llllllllll}
\hline$\Delta$ mag & $<0.05$ & 0.10 & 0.15 & 0.20 & 0.25 & 0.30 & 0.35 & 0.40 & 0.45 \\
Number & 32 & 29 & 20 & 10 & 9 & 10 & 5 & 2 & 2 \\
\hline
\end{tabular}

Table 3 which reports data for 119 pairs of consecutive days, taken from our 5 year long historical light curve: the median difference is just $0.1 \mathrm{mag}$. One might argue therefore that the IDV is due to a process different from that responsible for the long timescale variability of the source.

To further check this point we looked in our historical database and defined as a 'flare' any part of the light curve longer than three days limited by a local maximum and a local minimum, with amplitude larger than 0.3 mag. Both rising and falling branches of the light curve were considered. We found no correlation between the amplitude of a 'flare' and its average variation rate: in most cases $(2 / 3)$ this is less than $0.05 \mathrm{mag} / \mathrm{day}$ and in the remaining $1 / 3$ between 0.05 and $0.1 \mathrm{mag} / \mathrm{day}(0.004 \mathrm{mag} / \mathrm{hour})$. These values are definitely smaller than those found in the intranight variations, supporting the feeling that a different mechanism is responsible for the long term variations. 
A plot of the flare amplitude vs the log of its duration is given in Figure 2, lower right panel, together with two best-fitting straight lines: the flatter one (slope $=0.46$, $\mathrm{rms}=0.20)$ has $\log ($ time $)$ as the independent variable, the steeper one (slope $=1.19, \mathrm{rms}=0.27$ ) has $\Delta \mathrm{mag}$ as the independent variable. The amplitude of a 'flare' looks roughly related to its length, but the scatter of the data is rather large (the correlation coefficient is 0.63); a physical interpretation of this finding is not straightforward and beyond the scope of this paper.

\section{References}

Ghisellini, G., Padovani, P., Celotti, A., \& Maraschi, L. 1993, ApJ, 407, 65

Ghisellini, G., et al. 1997, A\&A, 327, 61
Giommi, P., et al. 1999, A\&A, 351, 59

Heidt, J., \& Wagner, S. J. 1996, AJ, 111, 2187

Nesci, R., Massaro, E., Maesano, M., Montagni, F., D’Alessio, F., Tosti, G., \& Luciani, M. 1998, IAU Symposium 188, eds Katsuji Koyama, Shunji Kitamoto, \& Masayuki Itoh (Dordrecht: Kluwer Academic), 442

Raiteri, C. M., Villata, M., \& Takalo, L. O. 1999, in Blazar Monitoring Towards the Third Millennium, Torino 1999, eds C. M. Raiteri, M. Villata, \& L. Takalo (Pino Torinese: Osservatorio Astronomico di Torino), 76

Villata, M., et al. 2000, A\&A, 363, 108 